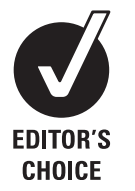

${ }^{1}$ Kerckhoff Heart Center, Department of Cardiology, Bad Nauheim, Germany

${ }^{2}$ Brigham and Women's

Hospital, Boston,

Massachusetts, USA

${ }^{3}$ Cleveland Clinic, Cleveland,

Ohio, USA

${ }^{4}$ Department of Neurology,

University of Western Australia,

Perth, Australia

${ }^{5}$ The Medicines Company, Zurich, Switzerland

${ }^{6}$ Department of Neurology, University of California, San Francisco, California, USA

${ }^{7}$ Institut de Cardiologie, Centre Hospitalier Universitaire

Pitié-Salpêtrière, Paris, France

${ }^{8}$ Mak Heart Clinic and Yong Loo Lin School of Medicine,

Singapore, China

${ }^{9}$ University and Royal Infirmary, Edinburgh, UK

${ }^{10}$ Department of Neurology,

Rhode Island Hospital and

Brown University, Providence,

Rhode Island, USA

${ }^{11}$ The Scripps Research Institute

and Scripps Clinic, La Jolla,

California, USA

\section{Correspondence to}

Dr Michael Weber, Kreisklinik

Gross-Umstadt, Department of

Cardiology, Krankenhaus

Strasse 11, 64823

Gross-Umstadt, Germany;

m.weber@kreiskliniken-dadi.de

Accepted 6 January 2011

Published Online First

10 March 2011

\title{
High-sensitivity C-reactive protein and clopidogrel treatment in patients at high risk of cardiovascular events: a substudy from the CHARISMA trial
}

\author{
Michael Weber, ${ }^{1}$ Deepak L Bhatt, ${ }^{2}$ Danielle M Brennan, ${ }^{3}$ Graeme J Hankey, ${ }^{4}$ \\ Steven R Steinhubl, ${ }^{5}$ S Claiborne Johnston, ${ }^{6}$ Gilles Montalescot, ${ }_{1}^{7}$ Koon-Hou Mak, ${ }^{8}$ \\ Keith A A Fox, ${ }^{9}$ Donald J Easton, ${ }^{10}$ Eric J Topol, ${ }^{11}$ Christian W Hamm, ${ }^{1}$ for the \\ CHARISMA Investigators
}

\begin{abstract}
Aims This study investigated the effect of clopidogrel treatment on inflammatory activity as evidenced by the change in high-sensitivity C-reactive protein (hsCRP) levels in a broad population of patients who are at high risk of atherothrombotic events. The predictive value of hsCRP levels for a treatment benefit of clopidogrel was also explored.
\end{abstract}

Methods The study included 8021 patients with established atherosclerotic disease or multiple cardiovascular risk factors enrolled in the CHARISMA trial. Patients were randomly assigned either to clopidogrel plus aspirin or placebo plus aspirin. HsCRP was measured at study entry and at study termination (median 28 months). The predefined primary composite endpoint was myocardial infarction, stroke, or death from cardiovascular causes.

Results There was a stepwise increase in the event rate of the combined primary endpoint with increasing quartiles of hsCRP at baseline $14.0 \%, 6.1 \%, 7.4 \%$ and $8.7 \%$ for the highest quartile). In both treatment groups the changes in hsCRP levels over time were identical. In patients with low hsCRP levels $(<3 \mathrm{mg} / \mathrm{l})$ clopidogrel treatment was associated with a lower event rate compared with placebo $(4.0 \%$ vs $6.0 \%$, log rank $\mathrm{p}=0.005)$. In contrast no treatment effect was observed in patients with high hsCRP levels $(8.1 \%$ vs $8.0 \%$, ns).

Conclusions In this broad population, hsCRP is a powerful predictor of ischaemic events. Compared with placebo, clopidogrel was without effect on inflammatory markers. The reduction in cardiovascular events by antiplatelet treatment with clopidogrel was isolated to patients with low levels of hsCRP.

Risk stratification of patients with acute coronary syndromes, stable coronary artery disease and patients with multiple cardiovascular risk factors is a major challenge and the basis for therapeutic decision-making. With a deeper understanding of the pathophysiological mechanisms leading to the initiation and progression of atherosclerotic plaques, inflammatory biomarkers, such as C-reactive protein (CRP), have gained great interest as prognostic tools for patients with suspected or overt coronary artery disease. ${ }^{1-3}$

In several large-scale studies it has consistently been demonstrated that levels of CRP, in particular high-sensitivity C-reactive protein (hsCRP), are closely linked to future adverse cardiac events in patients with an acute coronary syndrome, stable coronary heart disease and in apparently healthy

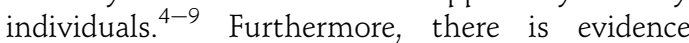
suggesting that lowering hsCRP levels by statin treatment may improve prognosis and, more importantly, that elevated hsCRP identifies patients who benefit from statin treatment independently from low-density lipoprotein (LDL) cholesterol levels. ${ }^{10} 11$

Clopidogrel treatment in combination with aspirin has been found to improve clinical outcome with a decrease in ischaemic events in patients with coronary heart disease. ${ }^{12-14}$ This beneficial effect is mainly thought to be related to the inhibition of the ADP-induced platelet aggregation by clopidogrel. However, recent data suggest that clopidogrel also has anti-inflammatory properties that might contribute to its beneficial effects. ${ }^{15} 16$

The objectives of the present substudy from the CHARISMA trial were thus threefold. First, to determine whether increasing serum levels of inflammatory markers, namely hsCRP, are associated with an increased risk of future vascular events, second whether clopidogrel attenuates inflammatory activity as evidenced by a decrease of inflammatory biomarkers (hsCRP), and third whether serum hsCRP levels at baseline identify patients who benefit from clopidogrel among those with clinically overt cardiovascular disease or multiple cardiovascular risk factors.

\section{METHODS}

\section{Trial design and patients}

The Clopidogrel for High Atherothrombotic Risk and Ischaemic Stabilisation, Management, and Avoidance (CHARISMA) trial was a prospective, multicentre, randomised, double-blind, placebo controlled study of the efficacy and safety of clopidogrel plus low-dose aspirin compared with aspirin alone in patients at high risk of a cardiovascular event. The details of the trial design and the results have been published previously. ${ }^{17} 18$ In brief, patients eligible for enrolment had to be at least 45 years of age and one of the following conditions had to be present: multiple cardiovascular risk factors or established cardiovascular disease, either documented coronary artery disease, documented cerebrovascular disease, or documented peripheral arterial disease. Exclusion criteria were oral antithrombotic medication, non-steroidal 
anti-inflammatory drugs, the need for clopidogrel treatment and scheduled revascularisation procedures.

After written informed consent, patients were randomly assigned either to clopidogrel plus aspirin or to placebo plus aspirin treatment. All patients received standard therapy at the discretion of the treating physicians. For this substudy 8021 patients, for whom samples for hsCRP measurement were available, were included and followed until study termination with a median duration of 28 months. The primary efficacy endpoint was the first occurrence of myocardial infarction, stroke of any cause or death from cardiovascular causes. The primary trial endpoints were adjudicated by the Cleveland Clinic clinical events committee, whose members were unaware of patients' treatment assignment.

\section{Laboratory assessment}

Venous blood samples were obtained at randomisation and at the last follow-up visit. Serum samples were frozen at $-80^{\circ} \mathrm{C}$ until analysis. HsCRP levels were determined by a chemiluminescent immunometric assay (Immulite; DPC, Los Angeles, USA) with a limit of quantification of $0.1 \mathrm{mg} / \mathrm{l}$, an intraassay coefficient of variation of $2.09 \%$, and an interassay coefficient of variation of $5.88 \%$.

\section{Statistical methods}

Continuous variables are presented as mean and SD if distributed normally or as median and IOR if not normally distributed. The distribution of the continuous variables was determined graphically by the use of histograms and normal probability plots. Categorical variables are presented as absolute numbers and percentages. Comparisons of continuous variables were performed using Student's t test (the Mann-Whitney U test was applied comparing hsCRP across treatment groups as hsCRP was not normally distributed). The $\chi^{2}$ test was used to compare categorical variables.

Multivariable models were constructed to assess the relationship of hsCRP and treatment to the primary efficacy outcome of cardiovascular death, myocardial infarction or stroke. HR and 95\% CI were calculated by Cox regression models and were adjusted for gender, age, body mass index, traditional risk factors-diabetes mellitus, hypertension, hypercholesterolaemia, current smoker and previous statin, angiotensin antagonist or ACE inhibitor medication, aspirin dose and evident cardiovascular disease versus multiple cardiovascular risk factors. As hsCRP was not normally distributed, it was entered into the multivariable model as a log-transformed variable. The HR is based on $1 \mathrm{SD}$ increment in the log of hsCRP. The interaction between the log of hsCRP and treatment was assessed. Additional analyses were performed by creating a cutpoint of the median hsCRP ( $3 \mathrm{mg} / \mathrm{dl})$. This was also used in multivariable modelling.

HsCRP levels were divided into quartiles. Comparisons of event rates across the quartiles were assessed. The treatment effect of clopidogrel was also explored per quartile by Kaplan-Meier estimates and log rank $p$ pvalues.

Kaplan-Meier time to event curves were plotted and estimates were tested using the log rank test. A $p$ value of less or equal to 0.05 was considered statistically significant. For all statistical analyses commercially available SAS statistical software was applied.

\section{RESULTS}

This substudy included 8021 patients (2550 women, mean age $64 \pm 9.5$ years) from the CHARISMA trial randomly assigned in equal proportions either to clopidogrel plus aspirin $(n=4002)$ or placebo plus aspirin $(n=4019)$. There were no differences between the study groups in baseline characteristics as shown in table 1. At the time of enrolment $5025(63 \%)$ patients were on statin treatment and 7427 (93\%) patients were already on aspirin treatment. Before enrolment 283 patients $(3.5 \%)$ were treated with either clopidogrel or ticlopidine. The baseline characteristics of the patients included in the present biomarker substudy did not differ from the baseline characteristics of the entire population of the CHARISMA trial. Approximately threequarters of the patients $(n=6141 ; 77 \%)$ had established atherosclerotic disease, whereas the remaining patients were included because of multiple cardiovascular risk factors. The prevalence of different kinds atherosclerotic disease as well as the prevalence of the cardiovascular risk factors are summarised in table 2 .

HsCRP measurements at study entry were available for 7534 (94\%) patients and at follow-up for 6568 (82\%) patients. HsCRP levels at study entry and at follow-up did not differ between patients randomly assigned to placebo or to clopidogrel (3.0 (1.4-6.5) mg/l vs $3.0(1.4-6.5) \mathrm{mg} / \mathrm{l} ; \mathrm{p}=0.86$, respectively, 2.8 $(1.4-6.1) \mathrm{mg} / \mathrm{l}$ vs $2.8(1.3-6.0) \mathrm{mg} / \mathrm{l} ; \mathrm{p}=0.39$, Mann-Whitney $\mathrm{U}$ test; figure 1). Relative changes in hsCRP levels from baseline to follow-up were not different between the two treatment groups $(0.24(-42.3-71.6) \%$ vs $-2.12(-41.4-64.7) \%$; $p=0.41$, respectively, Mann-Whitney U test).

The predefined composite primary endpoint was reached in 494 patients (6.2\%). There was a significant association of the primary endpoint and increasing quartiles of hsCRP (figure 2). As a continuous variable, the log-transformed hsCRP was univariately associated with the primary endpoint (HR per SD increment $1.31,95 \%$ CI 1.20 to 1.42 ). After adjustment for treatment, gender, age, body mass index, diabetes mellitus, hypertension, hypercholesterolaemia, current smoker, prevous statins, angiotensin antagonist or ACE inhibitor medication, aspirin dose and evident cardiovascular disease versus multiple cardiovascular risk factors, hsCRP remained statistically significant (HR per SD increment 1.31, 95\% CI 1.20 to 1.43; table 3). The interaction between the log of hsCRP and treatment group was not significant ( $p=0.25$, not shown). A similar association

Table 1 Baseline characteristics of the patients according to treatment group

\begin{tabular}{lll}
\hline & Treatment group & \\
\cline { 2 - 3 } & Placebo & Clopidogrel \\
\hline $\mathrm{N}$ & 4019 & 4002 \\
Age (years) & $64.3 \pm 9.6$ & $64.1 \pm 9.5$ \\
Gender (females, $\mathrm{n} / \%)$ & $1283(32 \%)$ & $1267(32 \%)$ \\
BMI $\left(\mathrm{kg} / \mathrm{m}^{2}\right)$ & $28.5 \pm 5.4$ & $28.7 \pm 5.3$ \\
Systolic blood pressure (mm Hg) & $140 \pm 20$ & $139 \pm 19$ \\
Heart rate (/min) & $71 \pm 12$ & $71 \pm 11$ \\
Cardiovascular risk factors & & \\
$\quad$ Hypertension (n/\%) & $3085(77 \%)$ & $3041(76 \%)$ \\
$\quad$ Hypercholesterolaemia (n/\%) & $2963(73 \%)$ & $2928(73 \%)$ \\
$\quad$ Diabetes mellitus (n/\%) & $1745(43 \%)$ & $1758(44 \%)$ \\
$\quad$ Current smoker (n/\%) & $506(13 \%)$ & $486(12 \%)$ \\
Previous medication & & \\
$\quad$ Statins (n/\%) & $2506(62 \%)$ & $2539(63 \%)$ \\
$\quad$ Fibrates (n/\%) & $251(6 \%)$ & $226(6 \%)$ \\
$\quad$ Aspirin (n/\%) & $3728(93 \%)$ & $3699(92 \%)$ \\
$\quad$ Thienopyridines (n/\%) & $142(4 \%)$ & $142(4 \%)$ \\
Platelets (10 $/ l)$ & $242 \pm 67$ & $242 \pm 66$ \\
hsCRP (mg/l) mean $\pm S D$ & $6.3 \pm 12.9$ & $6.4 \pm 12.5$ \\
hsCRP (mg/l) median (IOR) & $3.0(1.4-6.5)$ & $3.0(1.4-6.5)$ \\
\hline
\end{tabular}

BMI, body mass index; hsCRP, high-sensitivity C-reactive protein. 
Table 2 Inclusion criteria for patients with multiple atherothrombotic risk factors and for those with established cardiovascular disease

\begin{tabular}{lr}
\hline Clinically evident cardiovascular disease & \\
\hline Cerebrovascular disease & $696(12 \%)$ \\
$\quad$ Previous TIA & $1807(31 \%)$ \\
$\quad$ Previous ischaemic stroke & \\
Coronary heart disease & $720(12 \%)$ \\
$\quad$ Stable angina with documented CAD & $326(6 \%)$ \\
History of multivessel PCl & $581(10 \%)$ \\
History of multivessel CABG & $1399(24 \%)$ \\
Previous myocardial infarction & \\
Symptomatic peripheral arterial disease & $1003(23 \%)$ \\
Current intermittent claudication and ankle-brachial index $\leq 0.85$ & $857(17 \%)$ \\
$\quad$ History of intermittent claudication and previous intervention & \\
Multiple atherothrombotic risk factors & $1395(84 \%)$ \\
$\quad$ Type 1 or 2 diabetes (with drug therapy) & $745(57 \%)$ \\
Diabetic nephropathy & $89(25 \%)$ \\
Ankle-brachial index $<0.9$ & $115(22 \%)$ \\
Asymptomatic carotid stenosis $\geq 70 \%$ of luminal diameter & $208(49 \%)$ \\
$\geq 1$ Carotid plaque, as evidenced by intima-media thickness & $793(48 \%)$ \\
Systolic blood pressure $\geq 150$ mm $\mathrm{Hg}$, despite therapy for & \\
at least 3 months & $1086(71 \%)$ \\
Primary hypercholesterolaemia & $304(18 \%)$ \\
Current smoking $>15$ cigarettes/day & $815(48 \%)$ \\
Male sex and age $\geq 65$ years or female sex and age $\geq 70$ years &
\end{tabular}

CABG, coronary artery bypass grafting; $C A D$, coronary artery disease; $\mathrm{PCl}$, percutaneous coronary intervention; TIA, transient ischaemic attack.

was seen for each component of the primary endpoint (cardiovascular death, stroke, or myocardial infarction) evaluated separately. The relationship between hsCRP and clinical outcome was maintained even after adjusting for treatment group, gender, age, body mass index, diabetes mellitus, hypertension, hypercholesterolaemia, current smoker, previous statins, angiotensin antagonist or ACE inhibitor medication, aspirin dose and evident cardiovascular disease versus multiple cardiovascular risk factors (table 4). In contrast, no association

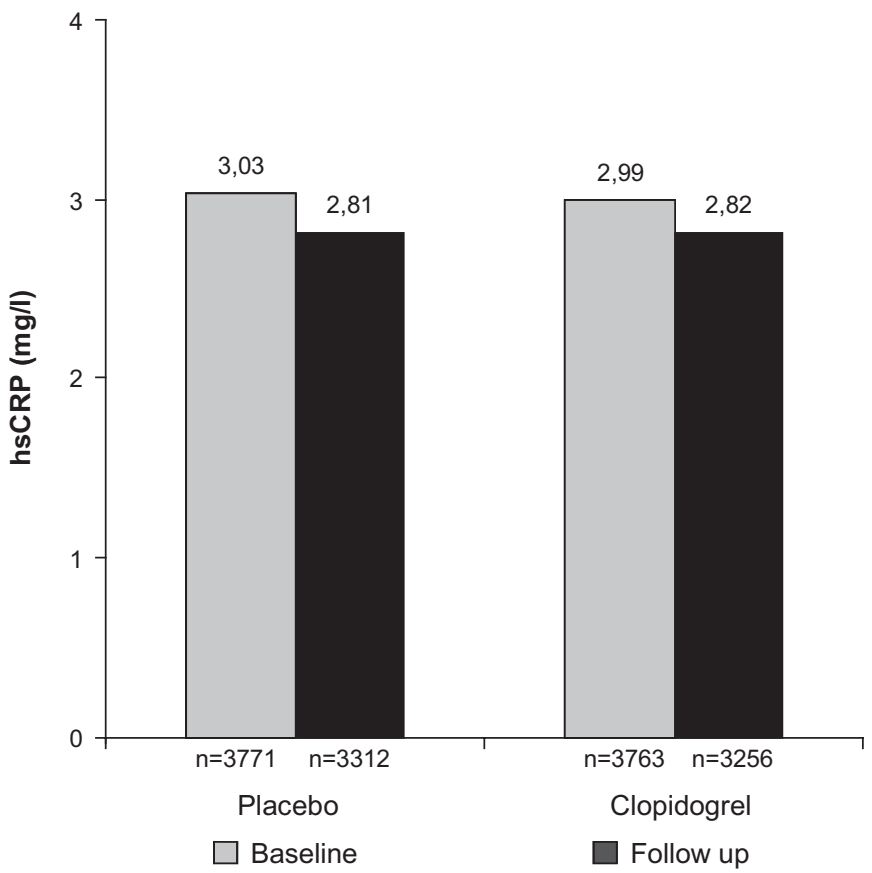

Figure 1 High-sensitivity C-reactive protein (hsCRP) levels at baseline (grey bars) and at follow-up (black bars) in relation to treatment. was present between hsCRP and any kind of bleeding events (data not shown).

In patients reaching the primary endpoint, hsCRP levels at baseline and at follow-up were higher compared with those without an event $(2.9 \mathrm{mg} / \mathrm{l}$ vs $4.0 \mathrm{mg} / \mathrm{l} ; \mathrm{p}<0.001$ at baseline and $2.8 \mathrm{mg} / \mathrm{l}$ vs $4.0 \mathrm{mg} / \mathrm{l} ; \mathrm{p}<0.001$ at follow-up). However, absolute and relative changes in hsCRP levels were identical in both groups.

Patients with hsCRP levels below the median $(<3.0 \mathrm{mg} / \mathrm{l})$ had a lower event rate compared with patients with high hsCRP levels $(5.0 \%$ vs $8.1 \%$; $<0.001)$. The rate of the primary endpoint was reduced with clopidogrel only in the group of patients with hsCRP below the median $(4.0 \%$ in the clopidogrel group vs $6.0 \%$ in the placebo group; $p<0.01$; figure 3 ). This difference in the frequency of the primary endpoint was driven by a lower stroke rate $(1.4 \%$ in the clopidogrel group vs $2.9 \%$ in the placebo group; $\mathrm{p}<0.01)$ and also a strong trend towards a lower rate of myocardial infarction (1.3\% in the clopidogrel group vs $2.4 \%$ in the placebo group; $p=0.058)$. Cardiovascular mortality was identical in both treatment groups $(1.6 \%$ in the placebo group vs $1.8 \%$ in the clopidogrel group; $\mathrm{p}=0.61$ ).

Even though patients with high hsCRP ( $\geq 3 \mathrm{mg} / \mathrm{l}$ ) levels had a significantly higher event rate than patients with hsCRP less than $3 \mathrm{mg} / \mathrm{l}$, the primary endpoint occurred at a similar rate in the two different treatment groups $(8.0 \%$ in the clopidogrel group vs $8.1 \%$ in the placebo group; $p=0.87$ ) and there was no difference for any individual component of the combined endpoint. The interaction between hsCRP (below and above $3 \mathrm{mg} / \mathrm{l}$ ) and treatment group was tested and found to be statistically significant $(p=0.036)$. After adjusting for gender, age, body mass index, diabetes mellitus, hypertension, hypercholesterolaemia, current smoker, previous statins, angiotensin antagonist or ACE inhibitor medication, aspirin dose and evident cardiovascular disease versus multiple cardiovascular risk factors, clopidogrel treatment was beneficial in patients with low hsCRP levels $(<3 \mathrm{mg} / \mathrm{l})$, whereas in patients with high hsCRP levels ( $\geq 3 \mathrm{mg} / \mathrm{l}$ ) clopidogrel treatment had no effect (table 5). Identical results could be obtained in the subgroup of patients with evident cardiovascular disease (data not shown).

\section{DISCUSSION}

In this substudy from the CHARISMA trial that includes a broad population of patients at high risk of cardiovascular events, we investigated the effect of clopidogrel on the

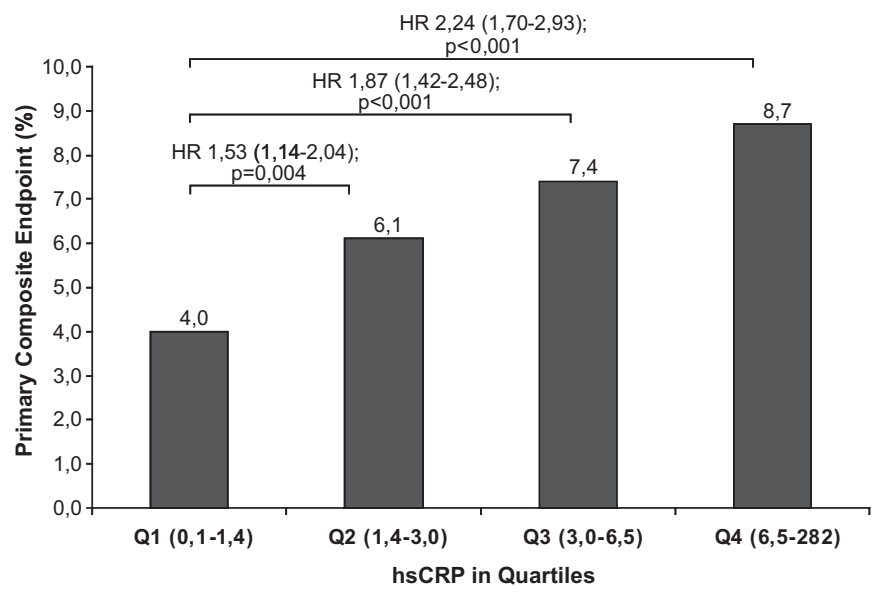

Figure 2 Event rate of the primary endpoint in relation to quartiles of high-sensitivity C-reactive protein (hsCRP) levels. 
Table 3 Multivariable model for the occurrence of the primary endpoint

\begin{tabular}{llrr}
\hline & HR 95\% CI & \multicolumn{1}{c}{$\boldsymbol{\chi}^{\mathbf{2}}$} & p Value \\
\hline Log transform of hsCRP, mg//* & $1.31(1.20$ to 1.43$)$ & 35.0 & $<0.001$ \\
Treatment group & $0.84(0.70$ to 0.99$)$ & 3.9 & 0.048 \\
Multiple risk factors vs CVD & $0.56(0.44$ to 0.73$)$ & 18.8 & $<0.001$ \\
Age, years & $1.05(1.04$ to 1.06$)$ & 84.2 & $<0.001$ \\
Male & $1.45(1.12$ to 1.78$)$ & 12.3 & 0.001 \\
BMI, $\mathrm{kg} / \mathrm{m}^{2}$ & $0.98(0.96$ to 0.99$)$ & 5.6 & 0.018 \\
Diabetes & $1.76(1.45$ to 2.14$)$ & 32.7 & $<0.001$ \\
Hypertension & $1.05(0.84$ to 1.3$)$ & 0.2 & 0.687 \\
Hypercholesterolaemia & $1.02(0.81$ to 1.28$)$ & 0.0 & 0.885 \\
Current smoker & $1.29(1.03$ to 1.62$)$ & 4.9 & 0.027 \\
Previous statins & $0.80(0.65$ to 0.99$)$ & 4.0 & 0.045 \\
Previous angiotensin II antagonists & $1.00(0.78$ to 1.28$)$ & 0.0 & 0.989 \\
Previous ramipril & $0.87(0.64$ to 1.2$)$ & 0.8 & 0.363 \\
Aspirin daily dose, mg & $1.00(1.00$ to 1.006$)$ & 3.8 & 0.052 \\
\hline
\end{tabular}

*Per 1 SD increment.

$\mathrm{BMI}$, body mass index; hsCRP, high-sensitivity C-reactive protein; CVD, cardiovascular disease.

inflammatory activity of atherosclerotic disease and the role of hsCRP as a predictor for a benefit from a treatment with clopidogrel in addition to aspirin.

This study has three major findings. First, hsCRP is a powerful predictor of ischaemic events. Second, compared with placebo, clopidogrel was without effect on inflammatory markers, and third, the reduction in cardiovascular events by antiplatelet treatment with clopidogrel was isolated to patients with low levels of hsCRP.

We confirmed that elevated levels of hsCRP are associated with an unfavourable clinical course. This finding is in line with the results from several reports demonstrating a predictive value of hsCRP in acute coronary syndromes, stable coronary artery disease, or in apparently healthy individuals. ${ }^{4-9}$ Of note, the cut-points of our per quartile analysis, $1.4 \mathrm{mg} / \mathrm{l}$ for the lowest quartile and $3.0 \mathrm{mg} / \mathrm{l}$ for the median, are almost identical with the cut-points for hsCRP recommended by the Centers for Disease Control and Prevention and the American Heart Association of less than $1 \mathrm{mg} / \mathrm{l}, 1-3 \mathrm{mg} / \mathrm{l}$ and more than $3 \mathrm{mg} / \mathrm{l}$ to categorise patients to be at low, average, or high risk. ${ }^{2} 19$

Even though the prognosis of patients with low hsCRP values was better compared with patients with high hsCRP levels, we observed an unexpected benefit from clopidogrel treatment only

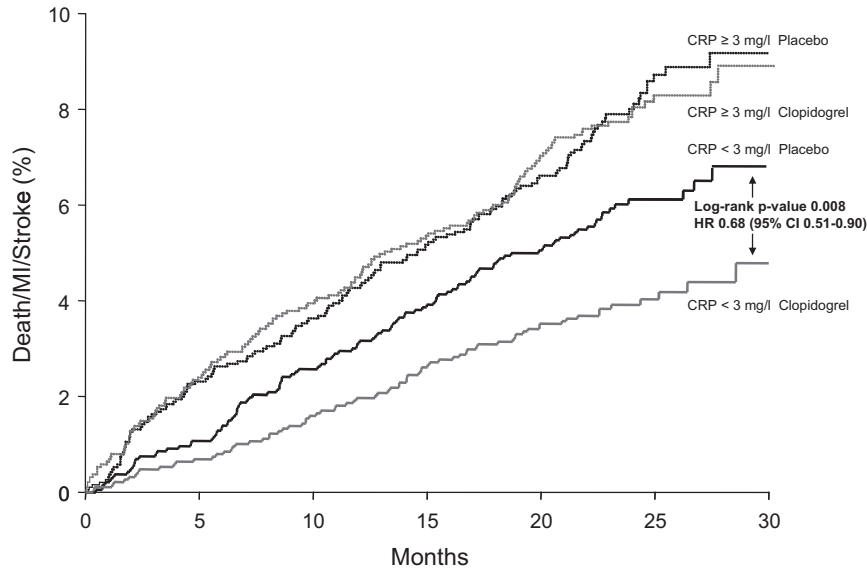

Figure 3 Kaplan-Meier curves for event-free survival according to high-sensitivity C-reactive protein (CRP) levels and treatment.

in this particular subgroup, with a decrease in the cardiovascular event rate in the active treatment arm. In contrast, no beneficial effect of clopidogrel was seen in patients with high levels of hsCRP. The favourable clinical outcome was mainly due to a reduction in stroke and a trend towards fewer myocardial infarctions. Cardiovascular mortality remained unaltered by clopidogrel treatment.

Several clinical and experimental studies have investigated the effect of clopidogrel on inflammatory activity in acute coronary syndromes, in stable coronary artery disease and in percutaneous coronary intervention with conflicting results. Whereas some studies found that hsCRP declined in clopidogrel and aspirintreated patients after an acute coronary syndrome, ${ }^{16}$ others did not find any effect of clopidogrel on inflammatory biomarkers. ${ }^{20} 21$ In the present study we did not detect an attenuation of inflammatory activity as evidenced by the inflammatory biomarker hsCRP in response to clopidogrel treatment. Therefore, our data suggest that an anti-inflammatory effect of clopidogrel may not be a key mechanism contributing to the beneficial effect of clopidogrel.

In contrast to the findings of a treatment benefit from clopidogrel in patients with low hsCRP, in the Physicians Health Study, the effect of aspirin was greater in patients with high hsCRP levels than in those patients with low levels. ${ }^{22}$ Aspirin is

Table 4 Multivariate Cox regression analysis for the occurrence of the primary endpoint

\begin{tabular}{|c|c|c|c|c|}
\hline & \multicolumn{4}{|l|}{ hsCRP } \\
\hline & $01(<1.4 \mathrm{mg} / \mathrm{l})$ & $02(1.4-3.0 \mathrm{mg} / \mathrm{l}$ & $03(3.0-4.5 \mathrm{mg} / \mathrm{l})$ & 04 (>4.5 mg/l) \\
\hline $\mathrm{N}$ & 1886 & 1871 & 1893 & 1884 \\
\hline \multicolumn{5}{|l|}{ Primary endpoint } \\
\hline$(n / \%)$ & $76(4.0 \%)$ & $114(6.1 \%)$ & $140(7.4 \%)$ & $164(8.7 \%)$ \\
\hline HR (95\% Cl) & & $1.5(1.1$ to 2.0$) ; p=0.004$ & 1.9 (1.4 to 2.5$) ; p<0.001$ & 2.2 (1.7 to 2.9 ); $p<0.001$ \\
\hline $\operatorname{HR}(95 \% \mathrm{Cl})^{*}$ & & $1.5(1.1$ to 2.1$) ; p=0.004$ & 2.0 (1.4 to 2.5 ); $p<0.001$ & 2.3 (1.7 to 3.0 ); $p<0.001$ \\
\hline \multicolumn{5}{|c|}{ Cardiovascular death } \\
\hline$(\mathrm{n} / \%)$ & $24(1.3 \%)$ & $38(2.0 \%)$ & $49(2.6 \%)$ & $80(4.2 \%)$ \\
\hline HR $(95 \%$ Cl) & & $1.6(0.96-2.7) ; p=0.068$ & $2.1(1.3-3.3) ; p=0.003$ & $3.4(2.2-5.4) ; p<0.001$ \\
\hline \multicolumn{5}{|l|}{ Stroke } \\
\hline$(\mathrm{n} / \%)$ & $28(1.5 \%)$ & $52(2.8 \%)$ & $58(3.1 \%)$ & $71(3.8 \%)$ \\
\hline HR (95\% Cl) & & $1.9(1.2$ to 3.0$) ; p=0.006$ & $2.1(1.3$ to 3.3$) ; p=0.001$ & 2.6 (1.7 to 4.0$) ; p<0.001$ \\
\hline \multicolumn{5}{|c|}{ Myocardial infarction } \\
\hline$(n / \%)$ & $29(1.5 \%)$ & $37(2.0 \%)$ & $65(3.4 \%)$ & $47(2.5 \%)$ \\
\hline HR (95\% Cl) & & $1.3(0.8$ to 2.1$) ; p=0.306$ & 2.3 (1.5 to 3.5$) p<0.001$ & 1.7 (1.05 to 2.6$) ; p=0.03$ \\
\hline
\end{tabular}

hsCRP, high-sensitivity C-reactive protein. 
Table 5 Frequency of the primary endpoint and its individual components in relation to quartiles of hsCRP

\begin{tabular}{lccc}
\hline & HR (95\% Cl)* & $\chi^{2}$ & p Value \\
\hline Clopidogrel vs placebo in hsCRP $<3 \mathrm{mg} / \mathrm{l}$ & $0.69(0.52$ to 0.93$)$ & 6.13 & 0.013 \\
Clopidogrel vs placebo in hsCRP $\geq 3 \mathrm{mg} / \mathrm{l}$ & $0.94(0.75$ to 1.18$)$ & 0.28 & 0.60 \\
Multiple risk factors vs CVD & $0.56(0.43$ to 0.72$)$ & 19.7 & $<0.001$ \\
Age, years & $1.05(1.04$ to 1.06$)$ & 84.61 & $<0.001$ \\
Male & $1.43(1.17$ to 1.76$)$ & 11.62 & 0.001 \\
BMI, kg/m & $0.98(0.96$ to 0.997$)$ & 5.30 & 0.02 \\
Diabetes & $1.76(1.45$ to 2.14$)$ & 32.82 & $<0.001$ \\
Hypertension & $1.04(0.83$ to 1.30$)$ & 0.09 & 0.76 \\
Hypercholesterolaemia & $1.02(0.81$ to 1.29$)$ & 0.03 & 0.87 \\
Current smoker & $1.31(1.05$ to 1.64$)$ & 5.48 & 0.019 \\
Previous statins & $0.79(0.64$ to 0.98$)$ & 4.52 & 0.034 \\
Previous angiotensin II antagonists & $1.01(0.79$ to 1.29$)$ & 0.003 & 0.95 \\
Previous ramipril & $0.86(0.64$ to 1.17$)$ & 0.94 & 0.33 \\
Aspirin daily dose, mg & $1.003(1.00$ to 1.01$)$ & 0.05
\end{tabular}

${ }^{*}$ Adjusted for treatment, gender, age, body mass index (BMI), diabetes mellitus, hypertension, hypercholesterolaemia, current smoker, previous statins, angiotensin antagonist or ACE inhibitor medication, aspirin dose and evident cardiovascular disease (CVD) versus multiple cardiovascular risk factors.

hsCRP, high-sensitivity C-reactive protein.

a non-selective inhibitor of the enzyme cyclooxygenase and thus poses both antiplatelet and anti-inflammatory properties, which could serve as an explanation for these contradictory results.

Our novel findings may inform the understanding of the underlying mechanisms leading to a progression of atherosclerosis and the occurrence of adverse cardiac events. In patients with low inflammatory activity the inhibition of platelet aggregation with the $\mathrm{ADP}$ receptor antagonist clopidogrel was effective, indicating a central role for platelet activation. In contrast, in patients with high inflammatory activity other mechanisms besides enhanced platelet activation seem to be of critical relevance.

Recent studies indicate that CRP is not a passive bystander but plays an active role in coronary artery disease, promoting atherosclerotic progression and inflammation. ${ }^{23}$ CRP inhibits endothelial nitric oxide synthase resulting in reduced nitric oxide release, and it stimulates endothelin 1, interleukin 6 and monocyte chemoattractant protein release as well as the expression of adhesion molecules. ${ }^{24}$ Enhanced inflammatory activity may thus dominate over the effects of platelet activation with respect to cardiovascular events in a stable population. In consequence, it seems reasonable that clopidogrel treatment may not be particularly effective in patients with high inflammatory activity in which platelet activation is only one element of a complex cascade of processes mediated by cytokines, chemokines and adhesion molecules leading to disease progression and events. In patients with low levels of inflammation, as measured by hsCRP, platelet activation becomes the determining factor for events. Here, platelet inhibition with clopidogrel may be particularly beneficial.

Several large-scale studies have evaluated the treatment effect of statins, which have anti-inflammatory properties in patients with acute coronary syndromes or stable coronary heart disease as well as in apparently healthy individuals. In these studies patients with elevated hsCRP levels benefited from statin treatment regardless of cholesterol and LDL-cholesterol levels. In contrast, patients without hsCRP elevation did not benefit. As a result of these studies, some have recommended the measurement of hsCRP to tailor statin treatment. ${ }^{10} 1125$

With the present data from the CHARISMA trial the relevance of hsCRP assessment for therapeutic decision-making may be supplemented by the recommendation that patients with established atherosclerotic disease or multiple cardiovascular risk factors and low hsCRP might derive benefit from treatment with clopidogrel.

However, as this is a retrospective analysis of a prospective study that has been conducted with a different aim, the results have to be interpreted with caution and should currently be considered as hypothesis generating, warranting further prospective evaluation.

\section{CONCLUSION}

The present data from the CHARISMA substudy demonstrate that the inflammatory biomarker (hsCRP) has prognostic value independent of traditional risk factors in predicting an adverse clinical course in patients with either overt atherosclerotic disease or multiple cardiovascular risk factors. Even though clopidogrel treatment had no impact on inflammation, low levels of hsCRP identified patients who benefited from clopidogrel treatment. Taken together, the results from the present study and from recent trials evaluating the predictive value of inflammatory markers for a treatment effect of statins, the data imply that hsCRP may be useful in optimising medical management. Accordingly, in patients with established atherosclerotic disease or multiple cardiovascular risk factors and low hsCRP values, dual anti-aggregatory therapy with clopidogrel plus aspirin may be considered. However, this recommendation needs further confirmation in prospectively designed studies.

Funding Funding for the CHARISMA trial was provided by Sanofi-Aventis and Bristol-Myers Squibb.

Competing interests DLB has received research grants (directly to the institution) from Bristol Myers Squibb, Sanofi Aventis; honoraria (currently donated to non-profits) from Astra Zeneca, Bristol Myers Squibb, Sanofi Aventis; speaker's bureau (not current, over 2 years ago) for Bristol Myers Squibb, Sanofi Aventis;

consultant/advisory board (currently donated to non-profits) for Astra Zeneca, Bristol Myers Squibb, Sanofi Aventis; expert testimony regarding clopidogrel (the compensation was donated to a non-profit organisation). GJH has received consulting fees from Sanofi-Aventis, Bristol-Myers Squibb, Bayer and Boehringer Ingelheim and lecture fees from Sanofi-Aventis, Bristol-Myers Squibb, Bayer and Boehringer Ingelheim. SRS has received consulting fees from Sanofi-Aventis, AstraZeneca, Eli Lilly and the Medicines Company. GM has received consulting and lecture fees from Sanofi-Aventis and Bristol-Myers Squibb. KAAF has received consulting fees from Sanofi-Aventis, lecture fees from Sanofi-Aventis and Bristol-Myers Squibb and grant support from Sanofi-Aventis. JDE has received consulting fees from Sanofi-Aventis 
and Bristol-Myers Squibb. EJT has served as a consultant to and has received lecture fees from Sanofi-Aventis and Bristol-Myers Squibb before 2005. CWH has received consulting and lecture fees from Sanofi-Aventis. MW, DMB, SCJ and K-HM declare no conflicts of interest.

Patient consent Obtained.

Ethics approval This study was conducted with the approval of the institutional ethics committee of each participating institution as well as the appropriate national ethics committees.

Contributors MW participated in the analysis of the data and the writing of the manuscript and has seen and approved the final version. DLB, GJH, SRS, SCJ, GM, K-HM, KAAF, JDE, EJT and CWH participated in the design and execution of the study, data analysis and review of the manuscript and have seen and approved the final version. DMB participated in the design of the study, analysis of the data and reviewing of the manuscript, and has seen and approved the final version.

Provenance and peer review Not commissioned; externally peer reviewed.

\section{REFERENCES}

1. Ballantyne CM, Nambi V. Markers of inflammation and their clinical significance. Atheroscler Suppl 2005;6:21-9.

2. Pearson TA, Mensah GA, Alexander RW, et al. Markers of inflammation and cardiovascular disease: application to clinical and public health practice. A statement for healthcare professionals from the Centers for Disease Control and Prevention and the American Heart Association. Circulation 2003:107:499-511.

3. Libby P, Theroux P. Pathophysiology of coronary artery disease. Circulation 2005:111:3481-8

4. Heeschen C, Hamm CW, Bruemmer J, et al. Predictive value of c-reactive protein and troponin $t$ in patients with unstable angina: a comparative analysis. Capture investigators. Chimeric c7e3 antiplatelet therapy in Unstable Angina Refractory to Standard Treatment trial. J Am Coll Cardiol 2000;35:1535-42.

5. James SK, Armstrong P, Barnathan E, et al. Troponin and C-reactive protein have different relations to subsequent mortality and myocardial infarction after acute coronary syndrome: a Gusto-IV substudy. J Am Coll Cardiol 2003;41:916-24.

6. Lindahl B, Toss H, Siegbahn A, et al. Markers of myocardial damage and inflammation in relation to long-term mortality in unstable coronary artery disease. FRISC Study Group. Fragmin during instability in coronary artery disease. N Engl J Med 2000;343:1139-47.

7. Schnabel R, Rupprecht HJ, Lackner KJ, et al. Analysis of N-terminal-pro-brain natriuretic peptide and $\mathrm{C}$-reactive protein for risk stratification in stable and unstable coronary artery disease: results from the Atherogene study. Eur Heart $J$ 2005; 26:241-9.

8. Ridker PM, Rifai N, Rose L, et al. Comparison of C-reactive protein and low-density lipoprotein cholesterol levels in the prediction of first cardiovascular events. $N$ Engl J Med 2002;347:1557-65.

9. Sabatine MS, Morrow DA, Jablonski KA, et al. Prognostic significance of the Centers for Disease Control/American Heart Association high-sensitivity C-reactive protein cut points for cardiovascular and other outcomes in patients with stable coronary artery disease. Circulation 2007:115:1528-36.

10. Ridker PM, Cannon CP, Morrow D, et al. C-reactive protein levels and outcomes after statin therapy. N Engl J Med 2005;352:20-8.

11. Ridker PM, Rifai N, Clearfield M, et al. Measurement of C-reactive protein for the targeting of statin therapy in the primary prevention of acute coronary events. N Engl $J$ Med 2001:344:1959-65.

12. Mehta SR, Yusuf S, Peters RJ, et al. Effects of pretreatment with clopidogrel and aspirin followed by long-term therapy in patients undergoing percutaneous coronary intervention: the PCl-CURE study. Lancet 2001;358:527-33.

13. Yusuf S, Zhao F, Mehta SR, et al; Clopidogrel in Unstable Angina to Prevent Recurrent Events Trial Investigators. Effects of clopidogrel in addition to aspirin in patients with acute coronary syndromes without ST-segment elevation. N Engl J Med 2001;344:494-502.

14. SteinhubI SR, Berger PB, Mann JT III, et al. Early and sustained dual oral antiplatelet therapy following percutaneous coronary intervention: a randomized controlled trial. JAMA 2002; 288:2411-20

15. Prasad K. C-reactive protein (CRP)-lowering agents. Cardiovasc Drug Rev 2006;24:33-50.

16. Woodward M, Lowe GD, Francis LM, et al. A randomized comparison of the effects of aspirin and clopidogrel on thrombotic risk factors and C-reactive protein following myocardial infarction: the Cadet trial. J Thromb Haemost 2004;2:1934-40.

17. Bhatt DL, Fox KA, Hacke W, et al. Clopidogrel and aspirin versus aspirin alone for the prevention of atherothrombotic events. N Engl J Med 2006;354:1706-17.

18. Bhatt DL, Flather MD, Hacke $W$, et al. Patients with prior myocardial infarction, stroke, or symptomatic peripheral arterial disease in the CHARISMA trial. J Am Coll Cardiol 2007;49:1982-8.

19. Morrow DA, Cannon CP, Jesse RL, et al. National Academy of Clinical Biochemistry laboratory medicine practice guidelines: clinical characteristics and utilization of biochemical markers in acute coronary syndromes. Circulation 2007:115:e356-75.

20. Azar RR, Kassab R, Zoghbi A, et al. Effects of clopidogrel on soluble CD40 ligand and on high-sensitivity C-reactive protein in patients with stable coronary artery disease. Am Heart J 2006:151:521

21. Montalescot G, Sideris G, Meuleman C, et al. A randomized comparison of high clopidogrel loading doses in patients with non-ST-segment elevation acute coronary syndromes: the ALBION (Assessment of the Best Loading Dose of Clopidogrel to Blunt Platelet Activation, Inflammation and Ongoing Necrosis) trial. J Am Coll Cardiol 2006:48:931-8.

22. Ridker PM, Cushman M, Stampfer MJ, et al. Inflammation, aspirin, and the risk of cardiovascular disease in apparently healthy men. $N$ Engl J Med 1997;336:973-9.

23. Szmitko PE, Wang $\mathrm{CH}$, Weisel RD, et al. New markers of inflammation and endothelial cell activation: Part I. Circulation 2003;108:1917-23.

24. Verma S, Wang CH, Li SH, et al. A self-fulfilling prophecy: C-reactive protein attenuates nitric oxide production and inhibits angiogenesis. Circulation 2002;106:913-19.

25. Ridker PM, Danielson E, Fonseca FA, et al. Rosuvastatin to prevent vascular events in men and women with elevated C-reactive protein. $N$ Engl J Med 2008:359:2195-207.

\section{Heart alerts}

Sign up for our electronic table of contents alert and you will never miss new issues of Heart when published online. Stay ahead and up to date by visiting heart.bmj.com. 